\title{
External quality assessment program for detection of glucose- 6-phosphate dehydrogenase deficiency in the Guangxi region
}

\author{
JUAN TANG ${ }^{1}$, XIANGYANG ZHOU ${ }^{1}$, XIAOCHUN LIU ${ }^{1}$, LEPING NING ${ }^{2}$, WEIYA ZHOU ${ }^{1}$ and YI HE ${ }^{1}$ \\ ${ }^{1}$ Clinical Laboratory Center and ${ }^{2}$ Department of Laboratory Medicine, The People's Hospital \\ of Guangxi Zhuang Autonomous Region, Nanning, Guangxi 530021, P.R. China
}

Received February 24, 2017; Accepted May 29, 2017

DOI: $10.3892 /$ etm.2017.4761

\begin{abstract}
The aim of this study is to improve the quality of testing for glucose-6-phosphate dehydrogenase (G6PD) deficiency through evaluation and analysis of the laboratory tests for G6PD activity. External quality assessment (EQA) was carried out twice per year with five samples each from 2014 to 2016. Samples were used for quantitative and qualitative assays. Quantitative results were collected, qualitative results were determined with reference values, and information about methods, reagents and instruments from participating laboratories within the required time. Laboratory performance scores, coefficient of variation $(\mathrm{CV})$, and the rates of false negative and positive results were calculated. As a result, a total of 2,834 cases of negative quality control (QC) samples and 2,451 cases of positive QC samples were assessed, where the rates of false negative and false positive results were $1.31 \%$ $(37 / 2,834)$ and $1.34 \%(33 / 2,451)$, respectively. Quantitative results indicated an increasing trend in testing quality, which were consistent with conclusions based on the comparison of EQA full-score and acceptable ratio in six assessments. The 2nd assay in 2016 had the best full-score ratio of $68.9 \%$ $(135 / 196)$ and best acceptable ratio of $84.2 \%$ (165/196). There was a decreasing trend in the average $\mathrm{CV}$ of six reagents produced in China, and the range of average $\mathrm{CV}$ increased to 14.6-23.6\% in 2016. The average CV of low level and high level samples was $22.5 \%$ and $15.3 \%$, respectively, demonstrating that samples with low G6PD activity have greater interlaboratory $\mathrm{CV}$ values. In conclusion, laboratories improved their testing quality and provided better diagnostic service for G6PD deficiency in areas with high incidence after participation in the EQA program in the Guangxi region.
\end{abstract}

Correspondence to: Dr Xiangyang Zhou, Clinical Laboratory Center, The People's Hospital of Guangxi Zhuang Autonomous Region, 6 Taoyuan Road, Nanning, Guangxi 530021, P.R. China E-mail: xfe9gp@163.com

Key words: external quality assessment, glucose-6-phosphate dehydrogenase, qualitative analysis, quantitative analysis

\section{Introduction}

Glucose-6-phosphate dehydrogenase (G6PD) deficiency is a common enzymopathic disease and is a single-gene disorder $(1,2)$. Previous studies indicated that there is a high frequency of this inherited blood disease among the population of southern China, and is primarily distributed in Guangxi, Guangdong, Yunnan, and the Sichuan region (3). A recent study showed that the incidence was $10.75 \%$ in the Guangxi region (4). G6PD deficiency is an incurable disease. However, population prevention, individual prevention and careful monitoring of neonates with this deficiency can prevent complications such as acute hemolytic anemia, jaundice, and kernicterus (5-7). It is imperative that large-scale screening for G6PD deficiency is performed, especially in pregnant women and newborns in areas with high incidence $(8,9)$.

A Guangxi external quality assessment (EQA) G6PD program was carried out in 2014. In this study, a 3-year EQA was carried out from 2014 to 2016 to describe the problems related to G6PD testing in the Guangxi region. Herein, we provide recommendations for corrective actions that can help reduce the error rate and decrease the interlaboratory coefficient of variation (CV), thus, ensuring consistent results between laboratories.

\section{Materials and methods}

Preparation of quality control samples for EQA. Anticoagulated whole blood from healthy donors [human immunodeficiency virus (HIV) antibodies, hepatitis $\mathrm{C}$ virus (HCV) antibodies, and hepatitis B surface antigen ( $\mathrm{HBsAg}$ ) were negative] was mixed and centrifuged to separate red blood cells (RBCs) and plasma. RBCs were washed three times with normal saline and stored at $-80^{\circ} \mathrm{C}$, followed by repeated freezing and thawing at $2-8^{\circ} \mathrm{C}$ to obtain hemolysates. After isolation of fibrinogen based on a methodo-logy using magnetic beads and filtration with 200-mesh screen filters, plasma was mixed with hemolysates as hemostroma and various doses of the G6PD analytical reagent to prepare high level and low level quality control (QC) samples. A total volume of $0.25 \mathrm{ml}$ of these samples were then dispensed into vials with a Hamilton dispenser and lyophilized with an LGJ-25C freeze dryer (Four-Ring Science Instrument Plant Beijing Co., Ltd, Beijing, China). 
Table I. Results of qualitative assays on G6PD from 2014 to 2016 in the Guangxi region.

\begin{tabular}{|c|c|c|c|c|c|}
\hline \multirow[b]{2}{*}{ Period } & \multirow[b]{2}{*}{ Assay } & \multicolumn{2}{|c|}{ Negative samples } & \multicolumn{2}{|c|}{ Positive samples } \\
\hline & & $\mathrm{n}$ & $\begin{array}{c}\text { False } \\
\text { negative }(\%)\end{array}$ & $\mathrm{n}$ & $\begin{array}{c}\text { False } \\
\text { positive }(\%)\end{array}$ \\
\hline \multirow[t]{2}{*}{2014} & 1st assay & 456 & $8(1.75)$ & 304 & $8(2.63)$ \\
\hline & 2nd assay & 310 & $8(2.58)$ & 465 & $8(1.72)$ \\
\hline \multirow[t]{2}{*}{2015} & 1st assay & 540 & $15(2.78)$ & 360 & $10(2.78)$ \\
\hline & 2nd assay & 364 & $5(1.37)$ & 546 & $4(0.73)$ \\
\hline \multirow[t]{2}{*}{2016} & 1st assay & 576 & $1(0.17)$ & 384 & $2(0.52)$ \\
\hline & 2nd assay & 588 & $0(0.00)$ & 392 & $1(0.26)$ \\
\hline Total & & 2,834 & $37(1.31)$ & 2,451 & $33(1.34)$ \\
\hline
\end{tabular}

G6PD, glucose-6-phosphate dehydrogenase.

Assessment of uniformity and stability of lyophilized QC samples. Ten samples were randomly chosen at each level for assessing the uniformity of dispensation and lyophilization. G6PD activity was determined monthly for 12 months to assess the stability of QC samples at $2-8^{\circ} \mathrm{C}$. The detection of G6PD activity and hemoglobin $(\mathrm{Hb})$ concentration were performed with a Beckman AU5800 chemistry analyzer (Beckman Coulter, Inc., Brea, CA, USA) and corresponding reagent kits (Changchun Huili Biotech Co., Ltd., China).

Design of external quality assessment. EQA (10) was performed twice per year (referred to as the 1st and 2nd assessments) with five QC samples each from 2014 to 2016, among which 2-3 vials were low level QC samples (1.0-2.5 U/gHb or 100-800 U/1), while the others were high level QC samples (7.0-17.0 U/gHb or 1700-2,400 U/1). Samples were then sequentially numbered and sent to participating laboratories. The ranges of G6PD activity for the different levels of QC samples were determined according to a cut-off value. A sample was considered to have G6PD deficiency if the value was $<4.8 \mathrm{U} / \mathrm{gHb}$ or $1,300 \mathrm{U} / 1$.

Treatment of QC samples. Lyophilized samples in vials were re-dissolved in $0.25 \mathrm{ml}$ of distilled water and allowed to stand for $30 \mathrm{~min}$. Samples were then mixed well by repeated inversion and processed according to the reagent instructions.

Implementation of external quality assessment. Data, including quantitative and qualitative results were requested to be returned via the internet within 7 days of completion. Quantitative data were filled correctly with units of $\mathrm{U} / \mathrm{gHb}$ or U/l, and qualitative data were expressed as positive (+) or negative (-) according to laboratory testing cut-off values. Negative meant normal whereas positive meant deficiency of G6PD. Data were analyzed based on the methods of detection and reagents used. Outliers, which are more than three standard deviations from the mean of a set, were eliminated after three repeated calculations. The median was considered as the reference value. Quantitative results that fell within two standard deviations from the reference value were permitted.
Evaluation of results. Data from participating laboratories were collected and analyzed with Microsoft Excel 2007 (Microsoft Corp., Redmond, WA, USA). For the quantitative survey, data within the permitted range was marked 20 points and considered acceptable if the total score was over 80 , while for the qualitative survey, outcomes corresponding with the expected results were marked 20 scores and the overall coincidence rates of samples were calculated.

\section{Results}

Results of confirmatory tests on lyophilized QC samples. There were no significant differences in G6PD activity of samples after being dispensed, lyophilized, and re-dissolved $(\mathrm{P}>0.05)$. Unused QC samples were stable for up to 1 year at $2-8^{\circ} \mathrm{C}$ and opened samples were stable for 3 days.

Collection of reports from participating laboratories. A total of 166 laboratories participated in the EQA program in 2014, while 152 and 155 effective reports were received for the 1st and 2 nd assessments, respectively, within the stated period following the distribution of QC samples. For surveys in 2015 and 2016, 185 and 196 laboratories were involved, while 180, 182, 192 and 196, effective reports were received, respectively. All participating laboratories performed the detection of G6PD activity with biochemistry analyzers and domestic reagents by the rate method (11). Among them, 25 laboratories determined the values of G6PD activity and hemoglobin concentration, while other laboratories analyzed only G6PD activity.

Results of qualitative assays. As shown in Table I, a total of 2,834 cases of negative QC samples and 2,451 cases of positive QC samples were assessed from 2014 to 2016, where the coincidence rate of negative and positive were $98.69 \%$ $(2,797 / 2,834)$ and $98.65 \%(2,418 / 2,451)$, respectively. The rate of false negative and false positive results were $1.31 \%$ $(37 / 2,834)$ and $1.34 \%(33 / 2,451)$, respectively. The best coincidence rate was found in 2016, where the rate of false negative and false positive results were $0 \%$ and $0.26 \%(1 / 392)$, respectively, on the 2nd assessment. 
Table II. Results of the EQA program for G6PD testing from 2014 to 2016 in the Guangxi region.

\begin{tabular}{|c|c|c|c|c|c|c|c|c|}
\hline \multirow[b]{2}{*}{ Period } & \multirow[b]{2}{*}{ Assay } & \multirow[b]{2}{*}{$\begin{array}{l}\text { Number of } \\
\text { laboratories }\end{array}$} & \multicolumn{6}{|c|}{ Results of EQA } \\
\hline & & & 100 scores & $\begin{array}{l}\text { Full-score } \\
\text { ratio }(\%)\end{array}$ & 80 scores & $\begin{array}{l}\text { Acceptable } \\
\text { ratio }(\%)\end{array}$ & $<80$ scores & $\begin{array}{c}\text { Reject } \\
\text { ratio }(\%)\end{array}$ \\
\hline \multirow[t]{2}{*}{2014} & 1st assay & 152 & 78 & 51.3 & 25 & 67.8 & 49 & 32.2 \\
\hline & 2nd assay & 155 & 84 & 54.2 & 24 & 69.7 & 47 & 30.3 \\
\hline \multirow[t]{2}{*}{2015} & 1st assay & 180 & 76 & 42.2 & 49 & 69.4 & 55 & 30.6 \\
\hline & 2nd assay & 182 & 115 & 63.2 & 35 & 82.4 & 32 & 17.6 \\
\hline \multirow[t]{2}{*}{2016} & 1st assay & 192 & 113 & 58.9 & 31 & 75.0 & 48 & 25.0 \\
\hline & 2nd assay & 196 & 135 & 68.9 & 30 & 84.2 & 31 & 15.8 \\
\hline
\end{tabular}

EQA, external quality assessment. G6PD, glucose-6-phosphate dehydrogenase.

Table III. Evaluation of the testing quality of different reagents.

\begin{tabular}{|c|c|c|c|c|c|c|c|c|c|c|c|c|}
\hline \multirow[b]{2}{*}{ Reagents } & \multicolumn{3}{|c|}{2014} & \multicolumn{3}{|c|}{2015} & \multicolumn{3}{|c|}{2016} & \multicolumn{3}{|c|}{ Total } \\
\hline & $\mathrm{N}$ & $\begin{array}{c}\text { Acceptable } \\
\text { ratio }(\%)\end{array}$ & $\begin{array}{l}\text { Average } \\
\mathrm{CV}(\%)\end{array}$ & $\mathrm{N}$ & $\begin{array}{c}\text { Acceptable } \\
\text { ratio }(\%)\end{array}$ & $\begin{array}{l}\text { Average } \\
\mathrm{CV}(\%)\end{array}$ & $\mathrm{N}$ & $\begin{array}{l}\text { Acceptable } \\
\text { ratio }(\%)\end{array}$ & $\begin{array}{l}\text { Average } \\
\mathrm{CV}(\%)\end{array}$ & $\mathrm{N}$ & $\begin{array}{c}\text { Acceptable } \\
\text { ratio }(\%)\end{array}$ & $\begin{array}{l}\text { Average } \\
\text { CV (\%) }\end{array}$ \\
\hline A & 16 & 82.3 & 33.7 & 23 & 80.6 & 24.6 & 25 & 89.3 & 18.8 & 64 & 84.1 & 25.7 \\
\hline B & 60 & 75.2 & 26.0 & 84 & 71.9 & 27.3 & 83 & 80.2 & 23.6 & 227 & 75.8 & 25.6 \\
\hline $\mathrm{C}$ & 39 & 93.2 & 20.4 & 41 & 92.6 & 15.4 & 41 & 94.1 & 14.6 & 121 & 93.3 & 16.8 \\
\hline $\mathrm{D}$ & 16 & 75.8 & 22.9 & 14 & 76.9 & 16.7 & 16 & 89.8 & 20.2 & 46 & 80.8 & 19.9 \\
\hline $\mathrm{E}$ & - & - & - & 13 & 76.2 & 15.7 & 16 & 84.2 & 15.4 & 29 & 80.2 & 15.6 \\
\hline $\mathrm{F}$ & - & - & - & 12 & 63.6 & 28.1 & 10 & 79.4 & 22.2 & 22 & 71.5 & 25.2 \\
\hline
\end{tabular}

$\mathrm{N}$ is the number of participating laboratories. $\mathrm{CV}$, coefficient of variation.

Table IV. Average CV of QC samples with different concentrations.

\begin{tabular}{lcccc}
\hline QC samples & Concentration & Unit & $\begin{array}{c}\text { Number of } \\
\text { laboratories }\end{array}$ & $\begin{array}{c}\text { Average } \\
\text { CV }(\%)\end{array}$ \\
\hline Low level & $1.0-2.0$ & $\mathrm{U} / \mathrm{gHb}$ & 182 & 27.7 \\
& $2.0-2.5$ & $\mathrm{U} / \mathrm{gHb}$ & 129 & 16.4 \\
& $100-400$ & $\mathrm{U} / 1$ & 1,507 & 26.3 \\
& $400-800$ & $\mathrm{U} / 1$ & 1,167 & 19.9 \\
High level & $7.0-10.0$ & $\mathrm{U} / \mathrm{gHb}$ & 200 & 15.4 \\
& $10.0-17.0$ & $\mathrm{U} / \mathrm{gHb}$ & 111 & 14.2 \\
& $1,700-2,400$ & $\mathrm{U} / 1$ & 1,481 & 16.4 \\
& & & & $(15.3)$ \\
\hline
\end{tabular}

Cut-off: $4.8 \mathrm{U} / \mathrm{gHb}$ and 1,300 U/1. CV, coefficient of variation; QC, quality control; $\mathrm{Hb}$, hemoglobin.

Results of quantitative assays. We compared the full-score ratio and acceptable ratio of the EQA program for G6PD testing performed in 2014, 2015 and 2016. Table II indicates that the 2nd assay in 2016 had the best results for full-score ratio $(68.9 \%, 135 / 196)$ and acceptable ratio $(84.2 \%, 165 / 196)$, while the 1st assay in 2015 had the lowest full-score ratio of $42.2 \%(76 / 180)$ and acceptable ratio of $69.4 \%(125 / 180)$, which were consistent with the results of the qualitative assay. Table III shows an increasing trend in the testing quality of six reagents produced in China, as the range of average CVs of different reagents increased to $14.6-23.6 \%$ in 2016, which was significantly lower than that in 2014 and 2015. Reagent B was the most commonly used, and was adopted in 227 laboratories. Reagent $\mathrm{C}$ had the best results of acceptable ratio (93.3\%) and average CV (16.8\%). Table IV demonstrates that samples with low G6PD activity had greater interlaboratory $\mathrm{CV}$ values. In this study, the average $\mathrm{CV}$ of low level $(1.0-2.5 \mathrm{U} / \mathrm{gHb}$ or $100-800 \mathrm{U} / 1)$ and high level (7.0-17.0 U/gHb or 1700-2400 U/l) QC samples was 22.5 and $15.3 \%$, respectively.

\section{Discussion}

This EQA program plays an instrumental role in monitoring and promoting the performance of G6PD testing in the Guangxi region. The coincidence rate of screening tests of QC samples for G6PD deficiency was relatively good. About $3 \%$ of reports were found to have false negative or positive results. False results were mainly attributed to clerical error or systematic error that made measurements consistently greater or lower than the true values. 
Data from the six EQA quantitative assays from 2014 to 2016 indicated a gradual improvement in the quality of G6PD tests. It was found that the acceptable ratio of the 1st assay in 2015 was lower than that of 2014, and this correlated with the additional reagents $\mathrm{E}$ and $\mathrm{F}$ used in tests and increasing $\mathrm{CV}$. Further investigation showed that this problem was principally attributed to improper procedure (incorrect handling of QC materials and reagents) and changes of operating process. Measurements were consistently higher than the true values since labeled values on the instructions were taken as the calibration value for the use of RANDOX QC materials. The results were lower than others obtained from tests with the same reagent because of the use of whole blood instead of hematocrit, as was required for dilution and detection. Additionally, G6PD reagents were not used with the corresponding $\mathrm{Hb}$ reagents for the measurement of G6PD in $\mathrm{Hb}$. For example, detecting G6PD activity with reagents provided by A while measuring $\mathrm{Hb}$ concentrations with reagents provided by other manufacturers would lead to large bias in the results. With the implementation of standard procedures, summary reports have been published on the website of the Guangxi Center for Clinical Laboratory (http://www.gxccl.com) as a warning and prevention to other laboratories. There was a decrease of the average $\mathrm{CV}$ and an increase of the acceptable ratio of all reagents in 2016.

Measurements of samples with different concentrations demonstrate that low level QC samples ranging from 1.0-2.0 U/gHb and 100-500 U/1 had large interlaboratory CV values $(27.7 \%$ and $26.3 \%$, respectively) while high level QC samples ranging from 7.0-17.0 U/g Hb and $>1,300 \mathrm{U} / 1 \mathrm{had}$ small CV values (the average CV was $15.3 \%$ ). This conclusion is consistent with recently published EQA data from the Taiwan region, which showed that the CV of samples with low G6PD activity were greater than those with high G6PD activity (12).

QC samples with critical G6PD activity were not evaluated in the present EQA program in the Guangxi region. Two critical values for G6PD activity in QC samples for EQA programs were reported by the College of American pathologists (CAP) in 2006 and 2007. The average values were 3.86 and $4.18 \mathrm{U} / \mathrm{gHb}$ (values ranged from $2.3-5.3 \mathrm{U} / \mathrm{gHb}$ and 1.8-6.7 $\mathrm{U} / \mathrm{gHb}$, respectively). The qualitative results $\mathrm{did}$ not meet the requirements of a coincidence rate $>90 \%$, and there was no comment on critical value samples from the CAP. Regarding assays in the Taiwan region, almost all interlaboratory CVs were controlled within $10 \%$ of QC samples with critical G6PD activity ranging from 3.0-4.3 $\mathrm{U} / \mathrm{gHb}$ and 4.4-6.0 U/g Hb (13). This has contributed to longstanding external quality assurance.

At present, a total of 197 laboratories have participated in the EQA program for G6PD testing in the Guangxi region, among which 95 implemented internal quality control, while 102 did not establish and execute internal quality control properly. Therefore, it is imperative for laboratories to strictly implement internal quality control (14). Participation in the
EQA program for G6PD testing is helpful for minimizing laboratory errors and improving testing quality, thus providing better diagnostic service for G6PD deficiency in areas with high incidence of the disease.

\section{Acknowledgements}

This study was supported by the Guangxi Health and Family Planning Commission Foundation (Z2012321).

\section{References}

1. von Seidlein L, Auburn S, Espino F, Shanks D, Cheng Q, McCarthy J, Baird K, Moyes C, Howes R, Ménard D, et al: Review of key knowledge gaps in glucose-6-phosphate dehydrogenase deficiency detection with regard to the safe clinical deployment of 8-aminoquinoline treatment regimens: A workshop report. Malar J 12: 112, 2013

2. Beutler E: Glucose-6-phosphate dehydrogenase deficiency: A historical perspective. Blood 111: 16-24, 2008.

3. Liu WL, Li F, He ZX, Jiang HY and Ai R: Identification of a case of glucose-6-phosphate dehydrogenase deficiency with G6PD Mediterranean-Middle East subtype in China. Int J Lab Hematol 35: e1-e3, 2013.

4. Yan T, Cai R, Mo O, Zhu D, Ouyang H, Huang L, Zhao M, Huang F, Li L, Liang $X$ and $\mathrm{Xu} X$ : Incidence and complete molecular characterization of glucose-6-phosphate dehydrogenase deficiency in the Guangxi Zhuang autonomous region of southern China: Description of four novel mutations. Haematologica 91: 1321-1328, 2006.

5. Elyassi AR and Rowshan HH: Perioperative management of the glucose-6-phosphate dehydrogenase deficient patient: A review of literature. Anesth Prog 56: 86-91, 2009.

6. Burton $\mathrm{C}$ and Kaczmarski R: Glucose 6-phosphate dehydrogenase deficiency and oxidative hemolysis. In: Clinical Studies in Medical Biochemistry. Glew RH and Rosenthal MD (eds). Vol 1. 3rd edition. Oxford University Press, New York, NY, pp123-133, 2007.

7. Tantular IS and Kawamoto F: An improved, simple screening method for detection of glucose-6-phosphate dehydrogenase deficiency. Trop Med Int Health 8: 569-574, 2003.

8. Al-Abdi SY, Mousa TA, Al-Aamri MA, Ul-Rahman NG and Abou-Mehrem AI: Hyperbilirubinemia in glucose-6-phosphate dehydrogenase-deficient male newborns in Al-Ahsa, Saudi Arabia. Saudi Med J 31: 175-179, 2010.

9. Al-Abdi SY, Alsaigh AS, Aldawoud FL and Al Sadiq AA: Lower reference limits of quantitative cord glucose-6-phosphate dehydrogenase estimated from healthy term neonates according to the Clinical and Laboratory Standards Institute guidelines: A cross sectional retrospective study. BMC Pediatr 13: 137, 2013.

10. International Organization for Standardization (ISO): ISO/IEC 17043: 2010, Conformity assessment - General requirements for proficiency testing. 1st edition. ISO, Geneva, pp1-39, 2010.

11. Roh ME, Oyet C, Orikiriza P, Wade M, Mwanga-Amumpaire J, Boum Y II, Kiwanuka GN and Parikh S: Screening for glucose-6-phosphate dehydrogenase deficiency using three detection methods: A cross-sectional survey in southwestern Uganda. Am J Trop Med Hyg 95: 1094-1099, 2016.

12. Chiang SH, Wu KF, Liu TT, Wu SJ and Hsiao KJ: Quality assurance program for neonatal screening of glucose-6-phosphate dehydrogenase deficiency. Southeast Asian J Trop Med Public Health 34 (Suppl 3): 130-134, 2003.

13. Chiang SH, Fan ML and Hsiao KJ: External quality assurance programme for newborn screening of glucose-6-phosphate dehydrogenase deficiency. Ann Acad Med Singapore 37 (Suppl): 84-4, 2008.

14. Eden PR and Herring CF III: Quality control in clinical laboratory samples. MLO Med Lab Obs 47: 22-23, 2015. 